Working Paper 8704

\begin{abstract}
A TECHNIQUE FOR ESTIMATING A COST
SYSTEM THAT ALOWS FOR INEFFICIENCY
\end{abstract}

by Paul W. Bauer

Paul W. Bauer is an economist at the Federal Reserve Bank of Cleveland.

Working papers of the Federal Reserve Bank of Cleveland are preliminary materials circulated to stimulate discussion and critical comment. The views stated herein are those of the author and not necessarily those of the Federal Reserve Bank of

Cleveland or of the Board of Governors of the Federal Reserve System.

May 1987 


\section{A TECHNIQUE FOR ESTIMATING A COST SYSTEM THAT ALOWS FOR INEFFICIENCY}

\section{Introduction}

The issue of how to estimate a system of cost and input share equations that allow for inefficiency has received increased attention in recent years. Techniques for estimating such systems have been proposed by Schmidt (1984), Melfi (1984), and Bauer (1985), but all of these techniques solve some problems at the expense of creating others. The central problem is how to relate the disturbances in the input share equations (some of which are the result of allocative inefficiency) to the allocative inefficiency term in the cost equation. With flexible, functional forms, such as the translog, no closed-form expression for this relation is possible. Schmidt, Melfi, and Bauer all tried to model this relationship in a feasible and qualitatively consistent manner, and each was successful to varying degrees.

This paper presents a new estimation technique that confronts this problem by using a collection of off-the-shelf techniques. The key to this new technique is to model the cost and input share equations in a qualitatively consistent fashion, but to avoid linking explicitly the disturbances in the input share equations to the disturbances in the cost equation. The likelihood function for such a system of cost and input share equations is derived by making the following distributional assumptions. The composed error term on the cost equation (composed of cost inefficiency and noise) is modeled as the sum of a truncated normal-random variable and a normal-random variable as proposed by Stevenson (1980). The disturbances in the input share 
equations are modeled as normal-random variables with nonzero means as suggested by Bauer (1985). Maximum likelihood estimates of the parameters of this model will be asymptotically efficient. An estimate of overall cost efficiency can then be obtained using a modified version of a technique developed by Jondrow, Love11, Materov, and Schmidt (1982) to decompose the residual on the cost equation into estimates of cost efficiency and noise. An algorithm proposed by Kopp and Diewert (1982) and improved by Zieschang (1983) can then be employed to decompose the estimate of cost efficiency into estimates of technical and allocative efficiency. An attractive procedure for estimating the cost frontier of an industry and for obtaining measures of technical and allocative efficiency for each firm in that industry over time is thus constructed.'

In this paper, the theory of the cost frontier and the Farrell measures of technical and allocative efficiency are reviewed in section II. Section III reviews the work of Schmidt, Melfi, and Bauer to lay the groundwork for the empirical technique that is proposed here. Section IV develops the empirical model and the steps required for its estimation, summarizing the relative advantages and disadvantages offered by this estimation technique. The conclusion is presented in section $V$.

\section{The Theory of Efficiency Measurement}

Economists routinely assume that firms try to minimize the cost of producing whatever level of outputs the firms' other behavioral goals deem optimal. The solution of the firm problem of cost minimization defines the cost function. The cost function has a number of desirable properties inherited from the underlying technology and the behavioral assumption of cost 
minimization (see Diewert [1974]). In logarithmic form, the cost function can be written as:

(1)

$$
\ln C^{*}=\ln C(y, w),
$$

where $C^{*}$ is the minimum cost of producing output vector $y \in R^{L}$ given input price vector $\boldsymbol{W E R}^{M}$, and where $L$ and $M$ are the number of outputs and inputs, respectively. Shephard's lemma can be invoked to obtain the cost-minimizing input share equations:

(2)

$$
\frac{\partial \ln C^{*}}{\partial \ln w_{i}}=s_{1}(y, w), \text { for } \mathbf{i}=1, \ldots, M \text {. }
$$

These functions describe the behavior of cost-efficient firms--the firms that operate on the cost frontier.

If a firm operates above the frontier, at higher-than-minimumcost, it is cost-inefficient. Farrell (1957) developed a measure of overall cost efficiency and then decomposed that measure into measures of technical efficiency (using proportionally too much of all inputs) and allocative efficiency (using the wrong mix of inputs). These efficiency measures are represented in figure 1, which illustrates the basic firm problem of cost minimization. The measure of overall cost efficiency is $E^{\circ}=0 a / o c$, which is the ratio of minimum (or efficient) cost to observed cost (using the definition of isocost curves). The measure of technical efficiency, given the above definition, is $E^{\top}=O b / o c$, which is the ratio of cost if the firm operated on the relevant isoquant (using the observed input mix) to observed cost. Finally, the measure of allocative efficiency is $E^{A}=0 a / o b$, the ratio of cost on the isoquant (using the observed input mix) to minimum cost. 
$-4-$

Figure 1 Cost-Minimization Problem

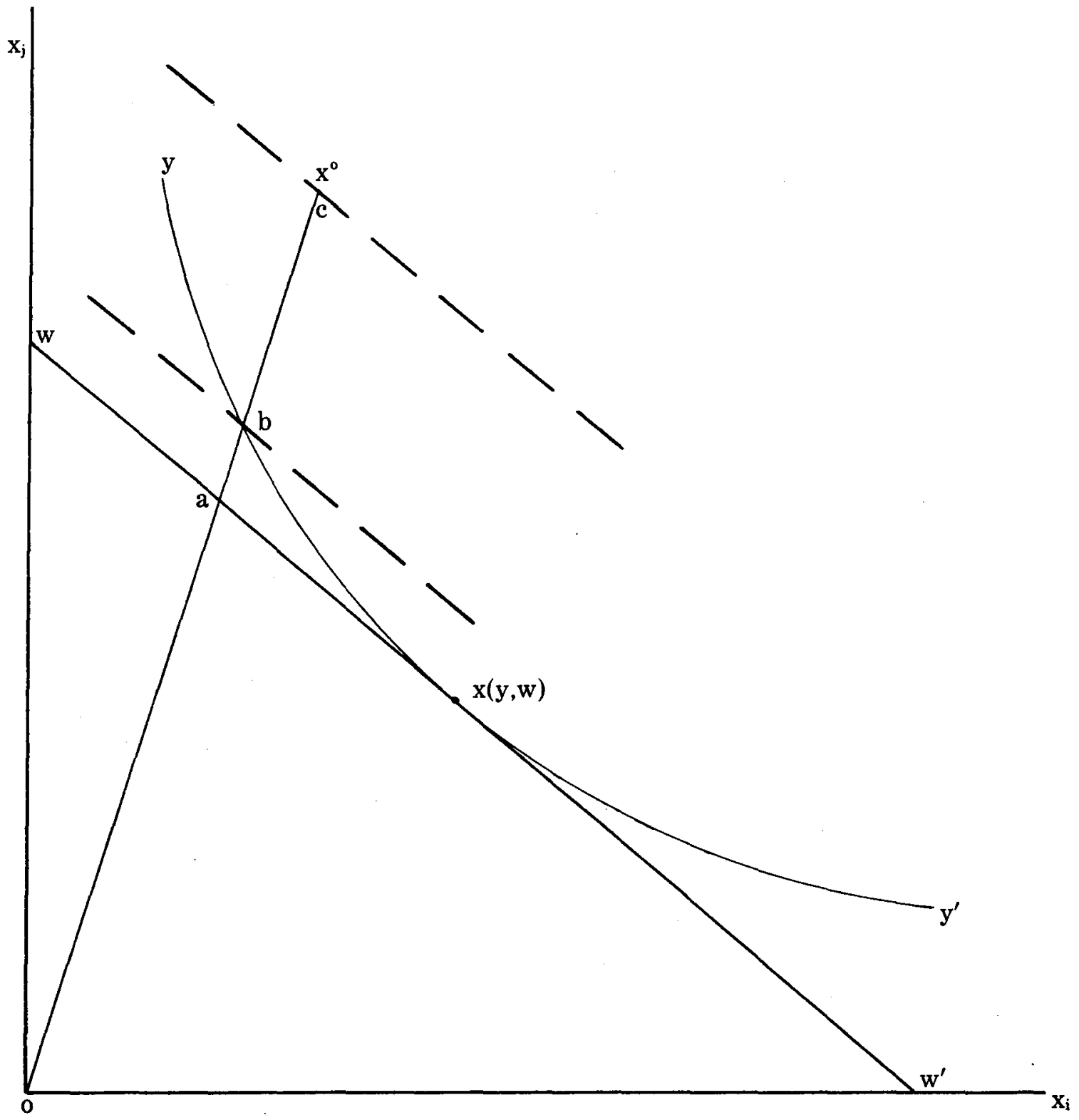


Note that $E^{O}=E^{\top} E^{A}$. These measures have the useful intrepretation that one minus any of the measures is the proportion by which costs could be lowered if that form of inefficiency were eliminated.

Observed cost can be written as follows using these definitions:

$$
\ln C=\ln C(y, w)+\ln T+\ln A,
$$

where $C$ is the observed cost, $\ln T$ is the increase in log cost due to technical inefficiency, and $\ln A$ is the increase in log cost due to allocative inefficiency. Thus, observed cost is equal to efficient cost plus the increase in cost because the firm is not technically and allocatively efficient. Note that the relationship between the efficiency measures and the above disturbances is:

$$
\begin{aligned}
& E^{\top}=e^{-\ln T} \text {, and } \\
& E^{A}=e^{-\ln A}
\end{aligned}
$$

A modified version of Shephard's lemma yields:

$$
\frac{\partial \ln C}{\partial \ln w_{i}}=s_{1}=s_{i}(y, w)+\frac{\partial \ln A}{\partial \ln w_{i}} \text {, for all } i=1, \ldots, M,
$$

where $s_{i}$ is the observed input share and $s_{1}(y, w)$ is the efficient input share. Thus, the observed input shares are equal to the efficient input shares plus a term that is related to the allocative inefficiency term in the cost equations. ${ }^{2}$ The problem of modeling this last term is often referred to as the Greene problem. The next section reviews the various attempts to model this term. 


\section{Survey of Existing Estimation Techniques}

In general, the cost sytem to be estimated can be written as:

(5)

$$
\begin{aligned}
\ln C_{n t} & =\operatorname{lnC}\left(y_{n t}, w_{n t}\right)+\operatorname{lnT} T_{n t}+\ln A_{n t}+v_{n t} \\
s_{1 n t} & =s_{i}\left(y_{n t}, w_{n t}\right)+\partial \ln A_{n t} / \partial \ln w_{i}+e_{i n t},
\end{aligned}
$$

where $v$ and e allow for noise on the cost and input share equations, respectively, and $n$ and $t$ refer to the $n$-th firm and the $t$-th time period, respectively.

The standard econometric technique for estimating a system of 'cost and input share equations in the absence of cost inefficiency was developed by Christensen and Greene (1976). Implicitly they assume that:

(6)

$$
\ln T_{n t}=\ln A_{n t}=\partial \ln A_{n t} / \partial \ln w_{i}=0 \text {, for all }(n, t) \text {. }
$$

Explicitly they assume that $\left(v_{n t}, e_{n t}\right)-N(0, \Sigma)$, a specification that allows for contemporaneous correlations among the disturbances. One input share equation must be dropped to avoid singularity of the system, but if iterative, seemingly unrelated regression (ITSUR) is employed, the estimates will not depend on which input share equation is dropped (see Barten [1969]). If one is interested only in estimating the average industry cost function and the errors are all interpreted as representing statistical noise, then this is a reasonable approach. This is also the appropriate approach if there are few degrees of freedom.

Nadiri and Schankerman (1981) point out that this approach may have problems if disturbances in the input share equations are regarded as 
àrising from "errors in optimization," as Christensen and Greene postulate (apparently using some notion of allocative inefficiency). The problem is that if the input share disturbances are the result of errors by the firm, then they should be related to the disturbance in the cost function. Schmidt (1984) puts it more succinctly: "Incorrect shares raise costs."

Schmidt (1984) was the first to propose a technique for estimating a cost system that allowed for inefficiency using flexible, functional forms. The key is how to model $\operatorname{dnA} / \partial \operatorname{lnw}_{\mathrm{i}}$. Schmidt and Lovell $(1979,1980)$ estimated a system of the production function and the first-order conditions for cost minimization using the Cobb-Douglas functional form and allowing for technical and allocative inefficiency, but their technique relies heavily on the self-dual nature of the Cobb-Douglas functional form. For flexible, functional forms, such as the translog, there exists no such closed-form solution to the dual problem, so one cannot obtain a closed-form expression for $\operatorname{alnA} / \partial \ln w_{1}$. Schmidt (1984) suggests the following as a convenient choice for modeling this unknown functional relationship between the input share disturbances and the allocative inefficiency term in the cost equation when using flexible, functional forms:

(7) $\quad \ln A_{n t}=\omega_{n t}{ }^{\prime} F \omega_{n t}$, where $\omega_{n t}=\nabla_{1 n w} \operatorname{lnA}$ and $F$ is a specified MxM positive semi-definite matrix. This specification has two desirable properties:

1. $\ln A_{n t} \geq 0$, with $\ln A_{n t}=0$ if and only if $\omega_{n t}=0$, and

2. $\quad \operatorname{nn} A_{n t}$ and $\left|\omega_{1, n t}\right|$ are positively correlated for all $\mathbf{i}$. 
The problem now is how to choose $F$. Schmidt assumes that $\omega-N(0, \Sigma)$ and sets $F=D^{1 /(N-1)} \Sigma^{+}$, where $D$ is the determinant of $\Sigma$ and $\Sigma^{+}$is the generalized inverse of $C$. These assumptions result in $1 n A_{n t}-X_{(M-1)}^{2}$ and $\ln A_{n t}$ and $\sigma_{i}^{2}$ being positively correlated for all $\mathbf{i}$.

The likelihood function for this model can be derived after positing an appropriate one-sided distribution for $\ln T_{n t}$ and letting $V_{n t}-N\left(0, \sigma_{v}^{2}\right)$; and asymptotically efficient maximum likelihood estimates for the parameters can be obtained. To date, no one has used this model empirically, presumably due to its relative intractability. The techniques developed by Melfi and Bauer, discussed below, are best viewed as attempts to derive a tractable empirical model using Schmidt's general approach.

Most of the complexity of Schmidt's model comes from the assumptions required to ensure that $1 n A_{n t}$ follows a known distribution. Melfi (1984) shows how the likelihood function for the system can be derived given the relation of the disturbances in the input share equations to the allocative inefficiency term in the cost equation by noting the following:

(8)

$$
f\left(\ln T_{n t}+\ln A_{n t}+V_{n t}\right)=g\left(\ln T_{n t}+V_{n t} \mid \omega_{n t}\right) h\left(\omega_{n t}\right),
$$

where $f, g$, and $h$ are the density functions for $\left(\ln T_{n t}+\ln A_{n t}+V_{n t}\right)$, $\left(1 n T_{n t}+v_{n t}\right)$, and $\omega_{n t}$, respectively. If $1 n A_{n t}$ is a function of $\omega_{n t}$, then $\ln A_{n t}$ is fixed given $\omega_{n t}$. Thus, distributional assumptions need to be made only concerning $\ln T_{n t}, v_{n t}$, and $\omega_{n t}$, which Melfi assumed to follow half-normal, normal, and multivariate-normal distributions, respectively. For tractability, Melfi modeled $F=I_{N}$. This seems to force the estimates of allocative inefficiency toward zero, since share deviations are less than one in absolute value and squaring them 
"shrinks" them.

Bauer (1985) made several modifications to Melfi's approach. First, allocative inefficiency is modeled as:

$$
\ln A_{n t}=\omega_{n t} \cdot F \omega_{n t}
$$

where, going back to Schmidt's original idea, $F$ is a positive, semidefinite matrix, but now the elements of $F$ are treated as parameters to be estimated. In practice, Bauer restricted $\mathrm{F}$ to be a diagonal matrix with nonnegative elements to reduce the dimensionality of the maximum likelihood problem. Bauer's second modification is to assume $\omega_{i n t} \sim N\left(\alpha_{i n}, \Sigma\right)$. Allowing $\omega_{i n t}$ to have a nonzero mean enables a given firm to over-or under-employ a given input over time--a proposition that seems highly plausible.

By the time of Bauer's analysis (1985), a fairly flexible technique for estimating a system of cost and input share equations in the presence of inefficiency had evolved. Inefficiency is modeled in a qualitatively consistent fashion, and the Greene problem is solved using a second-order quadratic function to model the relationship between $\omega_{n t}$ and $1 n A_{n t}$. This takes the Schmidt approach about as far as it can go; however, some problems remain. First, even for a small number of inputs and outputs, there are a large number of parameters to estimate. Some of these parameters, such as the off-diagonal elements of $F$ and 2, would be very difficult to estimate empirically without simplifying the model. ${ }^{3}$ More fundamentally, the use of an approximating function to relate $\omega_{n}$ to $1 n A_{n}$ may have conceptual problems. The most severe is that al though $\omega_{n t}$ is related to $1 n A_{n t}$ as described above, one cannot write the latter as a function of the former even if the cost function is homothetic (which is required to ensure that the level 
of output does not affect the measure of allocative inefficiency). This limits how accurately any approximating function of this type can track the true functional relationship. Consequently, using an approximation solves some problems at the expense of creating others, making empirical the issue of whether an approach that ignores the link among the inefficiency terms might yield better estimates.

\section{Proposed New Estimation Technique}

The new estimation technique is presented in this section. First, rewrite the cost equation in (5) as:

(10)

$$
1 n C_{n t}=\ln C\left(y_{n t}, w_{n t}\right)+u_{n t}+v_{n t} .
$$

The technical and allocative inefficiency terms $\left(\ln T_{n t}+\ln A_{n t}\right)$ are combined into the single term $u_{n t}$. Since no explicit relationship between the cost and input share equations is employed, modeling both technical and allocative inefficiency as a single, random variable in the cost equation simply acknowledges that both raise costs and that their effects cannot be separated using statistical means. Later it will be shown how estimates of the two types of cost inefficiency may be obtained using cost theory and the definitions of technical and allocative efficiency.

The inefficiency term, $\mathbf{u}_{\mathrm{n} t}$, follows a truncated-normal distribution with mode $\mu$ and underlying variance $\sigma_{v}^{2}$ such that $u_{n t} \geq 0$. The noise term, $v_{n t}$, is assumed to be independent of $u_{n t}$ and to follow a normal distribution with mean zero and variance $\sigma_{v}^{2}$. Stevenson (1980) derived the density function for the sum of a truncated-normal and a normal random 
variable $(\varepsilon=u+v)$ as:

(11)

$$
h(\varepsilon)=\sigma^{-1} f^{*} \frac{(\varepsilon-\mu)}{\sigma}\left[1-f^{*}\left(-\frac{\mu}{\sigma \lambda}-\frac{\varepsilon \lambda}{\sigma}\right)\right]\left[1-F^{*}\left(-\frac{\mu \mu}{\sigma_{u}}\right)\right]^{-1},
$$

where $\sigma=\left(\sigma_{u}^{2}+\sigma_{v}^{2}\right)^{1 / 2}, \lambda=\sigma_{v} / \sigma_{u}$, and $f^{*}(\bullet)$ and $F^{*}(\bullet)$ are the standard normal density and distribution functions, respectively.

All parameters of the model could be estimated using maximum likelihood estimation on just the cost equation, as proposed by Stevenson (19801, without making use of the input share equations at all. However, the input share equations contain additional information and increase the number of degrees of freedom in the system, so it would be inefficient in a statistical sense not to use them. The observed input share equations can be written as:

(12)

$$
s_{i n t}=s_{i}\left(y_{n t}, w_{n t}\right)+\frac{\partial \ln A}{\partial \ln W_{i}}+e_{i n t} \text {, for } i=1, \ldots, M-1 \text {. }
$$

Let $\mathbf{e}_{i n t}$ be normally distributed with mean zero and represent noise on the i- th input share equation. ${ }^{4}$

The term $\partial \ln A / \partial \ln w$, can be greater than, equal to, or less than zero depending on whether the $\mathrm{i}$-th input is over-or under-employed for a given firm at a particular time. Ideally, this term would be related to the inefficiency term in the cost equation, as the techniques described in the last section attempted to do. Given the problems discussed in the last section, it may be better to avoid trying to link the disturbances in the cost and input share equations.

The following is a theoretically consistent way to proceed given that the link between $u_{n t}$ and $\partial \ln A / \partial \ln w_{1}$ is neglected. Let $\partial \ln A / \partial \ln w_{1} \sim N\left(\alpha_{1}, \sigma_{\alpha_{1}}^{2}\right)$, where $\alpha_{i}$ is the tendency for firms to over-or under-employ the $\mathrm{i}$-th input 
over time. ${ }^{5}$ There is absolutely no reason to assume that $\boldsymbol{\alpha}_{1}=0$ a priori. ${ }^{6}$ The disturbance in the $\mathrm{i}-\mathrm{th}$ input share equation can be written as:

(13)

$$
\begin{aligned}
& \omega_{1 n t}=\left(\partial \ln A / \partial \ln w_{1}+e_{i n t}\right), \text { where } \\
& \omega_{i n t} \sim N\left(\alpha_{1}, \sigma_{\alpha i}^{2}+\sigma_{e i}^{2}\right), \text { or } \omega_{1 n t} \sim N\left(\alpha_{1}, \sigma_{\omega l}^{2}\right) \text {, and } \\
& \omega_{n t}=\left(\omega_{1 n t}, \ldots, \omega_{m-1, n t}\right)^{\prime} \sim N(\alpha, \Omega) .^{7}
\end{aligned}
$$

The complete cost system can now be written as:

$$
\begin{aligned}
& \ln C=\ln C(y, w)+u_{n t}+v_{n t} \\
& s_{\text {int }}=s_{i}(y, w)+w_{\text {int }}, i=1, \ldots, M-1 .
\end{aligned}
$$

The likelihood function for this system can be written as: ${ }^{8}$

$$
\begin{aligned}
& \operatorname{lnL}=-(T N M / 2) \ln 2 \pi-(T N / 2) \ln \sigma^{2}-(T N / 2) \ln |\Omega| \\
& -\left(1 / 2 \sigma^{2}\right) \sum_{t} \sum_{n}\left(\ln C_{n t}-\operatorname{lnC}\left(y_{n t}, W_{n t}\right)-\mu\right)^{2} \\
& +\sum_{t} \sum_{n} \ln \left[1-F^{*}\left(\sigma^{-1}\left(-\mu / \lambda-\left(\ln C_{n t}-\operatorname{lnC}\left(y_{n t}, W_{n t}\right)\right) \lambda\right)\right)\right] \\
& -(T N) \ln \left[1-F^{*}\left((-\mu / \sigma)\left(\lambda^{-2}+1\right)^{1 / 2}\right)\right] \\
& -(1 / 2) \sum_{t} \sum_{n}\left(\omega_{n t}-\alpha\right)^{\prime} \Omega^{-1}\left(\omega_{n t}-\alpha\right),
\end{aligned}
$$

where $\mathbf{t}=1, \ldots, \mathrm{T}$ and $\mathrm{n}=1, \ldots, \mathrm{N}$. Maximum likelihood estimates can be obtained for all the parameters in (15), and these estimates will be asymptotically efficient. A number of specification tests can be performed by using likelihood ratio tests similar to those proposed by Stevenson (1980).

While estimating the cost frontier is worthwhile and yields a great deal of valuable information about best-practice technology, estimates of $\ln T_{n t}$ and $I_{n} A_{n}$ yield valuable information about individual firm performance over time, which makes estimates of these terms worthwhile as well. The steps required to obtain estimates of these terms are now summarized. First, the 
work of Jondrow, Lovell, Materov, and Schmidt (1982) is modified to adjust for the estimation of a cost frontier (not a production frontier) and for the use of an inefficiency disturbance that is a truncated-normal (not a half-normal) random variabl'e. The conditional density of u given $\varepsilon=u+v$ is:

$$
\begin{gathered}
\text { (16) } f(u \mid \varepsilon)=1 /\left(2 \pi \sigma_{*}^{2}\right)^{1 / 2}[1-F(-\mu / \sigma \lambda-\varepsilon \lambda / \sigma)]^{-1} \exp \left[-\left(1 / 2 \sigma_{*}^{2}\right)\left(u-\left(\varepsilon \sigma_{u}^{2}+\mu \sigma_{v}^{2}\right) / \sigma^{2}\right)^{2}\right], \\
\text { for } u \geq 0 .
\end{gathered}
$$

which is just a $N\left(\left(\varepsilon \sigma_{u}^{2}+\mu \sigma_{v}^{2}\right) / \sigma^{2}, \sigma_{*}^{2}\right)$ truncated at zero, where $\sigma_{*}^{2}=\sigma_{u}^{2} \sigma_{v}^{2} / \sigma^{2} \cdot{ }^{g}$ Either the mode or the mean of this conditional distribution can be used as a point estimate of $u$ :

$$
M(u \mid \varepsilon)=\left(\varepsilon \sigma_{u}^{2}+\mu \sigma_{v}^{2}\right) / \sigma^{2} \text {, and }
$$

(18)

$$
E(u \mid \varepsilon)=\sigma_{*}\left[\frac{f\left(-\frac{\mu}{\sigma \lambda}-\frac{\varepsilon \lambda}{\sigma}\right)}{1-F\left(-\frac{\mu}{\sigma \lambda}-\frac{\varepsilon \lambda}{\sigma}\right)}-\left(\frac{\mu}{\sigma \lambda}+\frac{\varepsilon \lambda}{\sigma}\right)\right]
$$

The mode has the appealing interpretation of being the maximum likelihood estimator of $u$ given $E=U+v$ (see Materov [1981]). Note that, in practice, the terms required to compute $M(u \mid \varepsilon)$ and $E(u \mid \varepsilon)$ are unobserved and must be replaced by estimates of these parameters. Asymptotically, the measurement errors on all the terms disappear as the sample size is increased. However, $u$ would still be known imperfectly, since $\varepsilon$ contains only imperfect information about $u$.

Once an estimate of $u$ has been obtained, estimates of technical and allocative efficiency may be obtained using a technique proposed by Kopp and 
Diewert (1982) and improved upon by Zieschang (1983). Intuitively, given an estimate of overall cost efficiency and the cost frontier, and using duality theory, it should be possible to decompose the overall measure of cost efficiency into estimates of technical and allocative efficiency. Zieschang (1983) demonstrates how this can be done with a minimum of effort by solving the following set of nonlinear equations:

(19).

$$
\nabla_{w-} C\left(y, w^{-}, 1\right)-\nabla_{w M} C\left(y, w^{-}, 1\right) x^{*}=0
$$

where $w^{-}=\left(w_{1}, \ldots, w_{M-1}\right)^{\prime}$ and $x^{*}=\left(x_{1} / x_{M}, \ldots, x_{M-1} / x_{M}\right)^{\prime}$.

This is a system of $M-1$ equations in $M-1$ unknowns, $W^{-}$. If $W^{*-}$ solves the above system, then Zieschang has shown:

(20)

$$
E^{\top}=\nabla_{w M} C\left(y, w^{*-} /\left[\left(w^{*-}\right) \cdot x^{-}+x_{m}\right], 1\right) / x_{M},
$$

where $x^{-}=\left(x_{1}, \ldots, x_{m-1}\right)$. An estimate of $E^{A}$ can easily be obtained using the following:

(21)

$$
E^{A}=E^{O} / E^{\top} .
$$

Thus, estimates of $E^{\circ}, E_{n t}^{\top}$, and $E_{n t}^{A}$ can be obtained for each firm in each time period.

The advantages of this technique are that the likelihood function is more tractable, there are fewer parameters to estimate (since there is no equivalent to the $\mathbf{F}$ matrix to compute), and a less-arbitrary structure is imposed on the cost system. 
V. Conclusion

An econometric technique was developed for estimating a system of cost and input share equations by combining a collection of existing techniques in a new way. This technique provides a cleaner solution to the Greene problem than any currently in the literature by ignoring the relationship between the disturbances in the input share equation and the allocative inefficiency term in the cost equation, but modeling everything else in a qualitatively consistent fashion. Whether this technique outperforms those currently in the literature that do not ignore this relationship is an empirical question that will be explored in future research. This technique does appear to have the advantage of being easier to implement empirically than other proposed techniques. 


\section{$\underline{\text { Footnotes }}$}

1. Panel data is not required for estimation.

2. By definition, this term is not related to technical inefficiency, since technical efficiency is not a function of input prices.

3. Note that Bauer [1985] restricted both $F$ and $E$ to be diagonal matrices in obtaining his empirical estimates.

4. Again, one input share equation still must be dropped to avoid singularity of the system.

5. With panel data one could even allow $\alpha_{i n}$, so that the bias in input use could also vary from firm to firm.

6. In fact, Ferrier and Lovell (1987) have suggested estimating a translog system of cost and input share equations without the restriction that the intercept term in the input share equations be equal to the corresponding slope coefficiency in the cost equation as a test for the presence of allocative inefficiency.

7. It will not be possible to identify $\sigma_{\varepsilon ;}^{2}$ and $\sigma_{\alpha i}^{2}$, but these terms are of little interest in themselves.

8. Strictly speaking, it is incorrect to model the disturbances in the cost and input share equations as being independent, given the interdependence of $\partial \ln A_{n t} / \partial \ln w_{1 n t}$ and $u_{n t}$. However, as Schmidt (1984) pointed out, these terms will tend to be uncorrelated, since both negative and positive deviations from efficient shares raise costs. Thus, this specification may not be so far from the truth after all.

9. The firm and time subscripts can safely be omitted at this point for convenience. 
$\underline{\text { References }}$

Aigner, Dennis, C.A. Knox Lovell, and Peter Schmidt. "Formulation and Estimation of Stochastic Frontier Production Function Models," Journal of Econometrics, vol. 6, no. 1 (July 1977), 21-37.

Barten, A.P. "Maximum Likelihood Estimation of a Complete System of Demand Equations," European Economic Review, vol. 1, no. 1 (Fall 1969), 7-73.

Bauer, Paul W. "An Analysis of Multiproduct Technology and Efficiency Using the Joint Cost Function and Panel Data: An Application to the U.S. Airline Industry," Ph.D. dissertation, University of North Carolina at Chapel Hill, 1985.

Christensen., L.R... and W.R. Greene. "Economies of Scale in U.S. Electric Power Generation," Journal of Political Economy, vol. 84, no. 4, part 1, (1976), 655-76.

Diewert, W. Erwin. "Application of Duality Theory," in Frontiers of Quantitative Economics, vol. 2, M.D. Intrilligator and D.A. Kendrick, eds., Amsterdam: North-Holland Publishing Company, 1974, 106-71.

Fare, R., S. Grosskopf, and C.A. Knox Lovell. The Measurement Efficiency of Production, Boston: Kluwer-Nijhoff Publishers, 1985.

Farrell, M.J. "The Measurement of Productive Efficiency," Journal of Royal Statistical Society, Series A, General, vol. 120, part $3 \frac{(1957), 253-81 .}{.}$

Ferrier, Gary, and C.A. Knox Lovell. "Stochastic Cost Frontier Estimation," working paper, University of North Carolina at Chapel Hi11, 1987.

Forsund, Finn, C.A. Knox Lovell, and Peter Schmidt. "A Survey of Frontier Production Functions and of Their Relationship to Efficiency Measurement," Journal of Econometrics, vol. 13, no. 1 (May 1980), 5-25.

Fuss, M., and D. McFadden, eds. Production Economics: A Dual Approach to Theory and Applications, Amsterdam: North-Holland Publishing Company, 1978.

Greene, William H. "Maximum Likelihood Estimation of Econometric Frontier Functions," Journal of Econometrics, vol. 13, no. 1 (May 1980)[a], 27-56.

- "On the Estimation of a Flexible Frontier Production Model," Journal of Econometrics, vol. 13, no. 1 (May 1980)[b], 101-15.

Jondrow, James, C.A. Knox Lovell, Ivan S. Materov, and Peter Schmidt. "On the Estimation of Technical Inefficiency in the Stochastic Frontier Production Function Model," Journal of Econometrics, vol. 19, no. 2/3 (August 1982), 233-38.

Kopp, Raymond J. "The Measurement of Productive Efficiency: A Reconsideration," Quarterly Journal of Economics, vol. 96, no. 3 (August 1981), 477-503. 
and W. Erwin Diewert. "The Decomposition of Frontier Cost Function Deviations into Measures of Technical and Allocative Efficiency," Journal of Econometrics, vol. 19, no. 2/3 (August 1982), 319-31.

Materov, Ivan S. "On Full Identification of the Stochastic Production Frontier Model," Ekonomika i Matematicheskie Metody (Russian), vol. 17 (19811, 784-8.

Meeusen, Wim, and Julien van den Broeck. "Efficiency Estimation from Cobb-Douglas Production Functions with Composed Error," International Economic Review, vol. 18, no. 2 (June 19771, 435-44.

Melfi, C.A. "Estimation and Decomposition of Productive Efficiency in a Panel Data Model: An Application of Electric Utilities," Ph.D. dissertation, University of North Carolina at Chapel Hill, 1984.

Nadiri, Ishaq M., and M.A. Schankerman. "The Structure of Production, Technological Change and the Rate of Growth of Total Factor Productivity in the Bell System," in Productivity Measurement in Regulated Industries, Thomas G. Cowing and Rodney E. Stevenson, eds., New York: Academic Press, 1981, 219-47.

Pitt, Mark M., and Lung-Fei Lee. "The Measurement and Sources of Technical Inefficiency in the Indonesian Weaving Industry," Journal of Development Economics, vol. 9 (1981), 43-64.

Schmidt, Peter. "On the Statistical Estimation of Parametric Frontier Production Functions," Review of Economics and Statistics, vol. 58, no. 2 (May 1976), 238-39.

- "An Error Structure for Systems of Translog Cost and Share Equations," Econometrics Workshop Paper 8309, Department of Economics, Michigan State University, 1984.

, and C.A. Knox Lovell. "Estimating Technical and Allocative Inefficiency Relative to Stochastic Production and Cost Frontiers," Journal of Econometrics, vol. 9, no. 3 (February 19791, 343-66.

, and C.A. Knox Lovell. "Estimating Stochastic Production and Cost Frontiers When Technical and Allocative Inefficiency Are Correlated," Journal of Econometrics, vol. 13, no. 1 (May 19801, 83-100.

, and Robin C. Sickles. "Production Frontiers and Panel Data," Journal of Business and Economic Statistics, vol. 2, no. 4 (October 19841, 367-74.

Shephard, Ronald W. Theory of Cost and Production Functions, Princeton: Princeton University Press, 1970.

Waldman, Donald M. "Properties of Technical Efficiency Estimators in the Stochastic Frontier Model," Journal of Econometrics, vol. 25, no. 3 (July 19841, 353-64. 
Stevenson, Rodney E. "Likelihood Functions for Generalized Stochastic Frontier Estimation," Journal of Econometrics, vol. 13, no. 1 (1980), 57-66.

Zieschang, Kimberly D. "A Note on the Decomposition of Cost Efficiency into Technical and Allocative Components," Journal of Econometrics, vol. 23, no. 3 (December 1983), 401-5. 